\title{
Role of gadolinium-based contrast agents in neurological disorders
}

\author{
Weronika Golec ${ }^{1}$, Aleksandra Jakimów-Kostrzewa ${ }^{2}$, Bartosz Mruk ${ }^{3}$, Sergiusz Jóźwiak ${ }^{1}$ \\ ${ }^{1}$ Department of Child Neurology, Medical University of Warsaw, Warsaw, Poland \\ ${ }^{2}$ Department of Radiology, Medical University of Warsaw, Warsaw, Poland \\ ${ }^{3}$ Department of Radiology, Centre of Postgraduate Medical Education, Warsaw, Poland
}

\begin{abstract}
Gadolinium-based contrast agents (GBCAs) are widely used in magnetic resonance imaging (MRI) to help with the diagnostic and monitoring processes of many diseases, including neurological disorders. Initially, it was assumed that GBCAs carry minimal risk, are safe and well tolerated. But recent reports of GBCA-associated deposition in many body tissues have raised concerns about the broader health impacts of gadolinium exposure. The aim of this review was to summarise knowledge regarding gadolinium deposition, primarily in the brain structures, and of potential GBCA-associated toxicity. Moreover, we discuss the current recommendations on the use of GBCAs, as well as alternative contrast agents and imaging techniques.

Key words: gadolinium-based contrast agents, gadolinium deposition, alternative contrast agents, alternative imaging techniques
\end{abstract}

(Neurol Neurochir Pol 2020; 54 (5): 399-409)

\section{Introduction}

Magnetic resonance imaging (MRI) is one of the fastest developing imaging methods and has been widely used for diagnostic and monitoring purposes of many pathological conditions, including neurological disorders [1]. Introduced into clinical practice in the early 1990s, this method of neuroimaging quickly became commonly used in the assessment of inflammatory and demyelinating lesions, defects of the central nervous system (CNS), spinal cord, vascular diseases and proliferative processes [2].

Of all the benefits of MRI, the greatest are its high tissue resolution (where it is the best of all neuroimaging methods), its safety (no exposure to ionising radiation), and its non-invasiveness. Admittedly, MRI also has a few drawbacks, of which the most impactful are low spatial resolution, a long time of data acquisition, susceptibility to movement artifacts (the necessity of anaesthesia in paediatric patients), and difficulties in calcifications imaging [2]. To improve the quality of images, this technique has been refined over the years.

One of the most significant improvements in MRI has been the use of gadolinium-based contrast agents (GBCAs) [3].
They have long been considered to be highly effective and safe. However, recent reports on the retention of GBCAs in human tissue, mainly in brain structures, have called into question the safety of GBCA administration. Taken together, these reports have led to intensive research into MRI techniques which could replace the GBCA-enhanced study, into improving current imaging methods, and into seeking completely new contrast agents.

In this review, we discuss the current opinions on gadolinium deposition, mainly in brain structures, and review the literature on GBCA-associated toxicity, alternative imaging techniques, and contrast agents. Additionally, we summarise medical society recommendations and perspectives on the use of GBCAs in clinical practice.

\section{Methods}

A comprehensive literature search using the PubMed, Google Scholar, and Ovid Medline databases was conducted for published works regarding Gd deposition, mainly in the brain structures, GBCA-associated toxicity, alternative non-contrast-enhanced (NCE) techniques of MRI, new 
GBCAs, and alternative MRI contrast agents. We also reviewed the updated recommendations of the European Medicines Agency on GBCA usage.

\section{Characteristics of GBCAs}

In 1988, the first GBCA, gadopentetate dimeglumine (Magnevist ), enhanced MRI was performed. Since then, the U.S. Food and Drug Administration (FDA) has approved a further eight GBCAs for use (Tab. 1) [3]. All GBCAs contain the paramagnetic ion of the rare earth metal gadolinium $\left(\mathrm{Gd}^{3+}\right)$, which possesses the most unpaired electrons of any stable ion (seven), creating a high magnetic moment that can enhance proton relaxation [4].

GBCAs are positive agents that shorten the T1 (increase signal to longitudinal) relaxation time of surrounding water protons to indirectly produce a signal-enhancing effect [5]. In CNS diseases, contrast-enhancement MRI (CE-MRI) allows the visualisation of small lesions, the depiction of an impaired blood-brain barrier (BBB), and the revelation of vessel structures and of primary and secondary CNS tumours. It also provides information on the location and grade of lesions, treatment planning, and monitoring of responses to therapy $[3,6]$.

It is estimated that today GBCAs are used in more than one third of all MRI studies [7]. Every year, over 30 million doses of GBCAs are administered worldwide [8].

Free gadolinium, by competitive blocking of calcium channels, demonstrates strong cytotoxic capabilities which significantly disturb the functions of various body cells, including nerve cells. Gadolinium also impairs the activity of intracellular enzymes by displacing endogenous metals such as zinc and copper [9]. The use of gadolinium compounds as contrast agents has become possible by combining gadolinium ions with organic ligands, thereby forming chelating molecules. This leads to a reduction in the toxicity of GBCAs, their safe intravenous administration, and excretion in the bound form by the kidneys or bile [10]. GBCAs can be categorised in two ways due to their biochemical structure (linear and macrocyclic) and the total compound charge (ionic and nonionic) [11]. GBCA structure is important because it affects both the stability of the chelate and the side effects it causes. Studies indicate that macrocyclic compounds compared to linear are characterised by greater stability, which is associated with a greater ability to bind gadolinium ions [12].

\section{Side effects of GBCAs}

\section{Adverse reactions}

GBCAs are regarded as being safer than iodine contrast agents. The incidence of immediate allergic reactions after GBCA administration is low [13]. Immediate adverse reactions are hypersensitivity reactions which occur within an hour of exposure to GBCAs. According to their severity they can be further divided into mild, moderate, and severe reactions [14].

The adverse event rate for GBCAs ranges from $0.07 \%$ to $2.4 \%$ [15]. The most common adverse reactions are mild. They include coldness, warmth, or pain at the injection site, paresthesia, nausea, vomiting, headache and dizziness. Allergic-like reactions are uncommon and vary in frequency from $0.004 \%$ to $0.7 \%$ [15]. Severe, life-threatening anaphylactic reactions are extremely rare, ranging from $0.001 \%$ to $0.01 \%$ [15]. The strongest adverse reaction predictor factor is a history of an adverse reaction after previous GBCA administration [16]. A recently conducted meta-analysis correlating adverse reaction occurrence to properties of GBCAs indicates that the risk of immediate allergic reactions is based on the chemical structure, ionicity and affinity for serum proteins. It has been proven that ionic agents, serum protein binding agents and macrocyclic compounds increase the risk of immediate allergic-like reactions [17].

The risk of such a reaction is not related to the osmolality of the contrast agent: the low doses used make the osmolar load very small [18]. For the same reason, the risk of post-contrast acute kidney injury (PC-AKI) is very low when GBCAs are used [18]. There is also no difference in the incidence of acute adverse reactions among the gadolinium-based extracellular agents [18].

Table 1. Characteristics of FDA-approved GBCAs $[2,3]$

\begin{tabular}{lccc} 
Generic name & Brand name & Chemical structure & Elimination pathway \\
\hline Gadodiamide & Omniscan & Linear nonionic & Renal \\
Gadoversetamide & OptiMARK & Linear nonionic & Renal \\
Gadopentetate dimeglumine & Magnevist & Linear ionic & Renal \\
Gadobenate dimeglumine & MultiHance & Linear ionic & Renal; 4-5\% Biliary \\
Gadoxetic acid & Primovist & Linear ionic & $50 \%$ Renal; $50 \%$ Biliary \\
Gadofosveset trisodium & Ablavar/Vasovist & Linear ionic & Renal; 4-5\% Biliary \\
Gadoteridol & ProHance & Macrocyclic nonionic & Renal \\
Gadobutrol & Gadovist/Gadavist & Macrocyclic nonionic & Renal \\
Gadoterate meglumine & Dotarem & Macrocyclic ionic & Renal
\end{tabular}




\section{Evidence of gadolinium residues accumulation in human tissues}

The first description of potential gadolinium compound accumulation in animal tissues was published in 1995 [19]. Gibby et al. in 2003 indicated that GBCAs could accumulate in human bones. During hip arthroplasty procedures, the authors gathered samples of bone tissue from patients in whom gadodiamide (linear GBCA) or gadoterydol (macrocyclic GBCA) were used. The concentration of gadolinium compounds was 2.5 times higher in the former than in the latter patients [20]. In 2005, the use of Inductively Coupled Plasma - Mass Spectrometry (ICP-MS) confirmed the deposition of gadolinium in human bone tissue and showed a four-times higher concentration of gadolinium compound after gadodiamide administration compared to gadoterydol [21].

The toxicity of GBCAs was further confirmed in 2006 by Grobner et al. who linked multiple GBCA exposures in patients with renal dysfunction to nephrogenic systemic fibrosis (NSF) [22]. NSF is a rare, late adverse reaction associated with exposure to GBCAs, which can potentially be life-threatening and is characterised by fibrosis of the skin and potential systemic involvement of the joints, lungs, heart, liver, and muscles [22, 23]. The risk factors for NSF can be distinguished into a biochemical structure and stability of GBCAs and patient-related factors. NSF occurrence is strictly associated with the use of linear GBCAs. Gadodiamide, gadopentetate dimeglumine and gadoversetamide are associated with a high risk of NSF, and are currently suspended [23]. The main risk factor when considering NSF probability is renal function status. It has been proven that the risk of NSF is substantially higher in patients with acute kidney injury (AKI) or severe chronic kidney disease $(\mathrm{CKD})$ (estimated glomerular filtration rate [eGFR] $<30 \mathrm{~mL} / \mathrm{min} / 1.73 \mathrm{~m}^{2}$ ), and those on dialysis. It is crucial to prevent NSF, due to its incompletely understood mechanism and the absence of effective treatment. Therefore, it is recommended considering on a case-by-case basis the need for contrast-enhanced MRI in patients with severe CKD and who are receiving dialysis. Alternatives to an MRI diagnostic test should be investigated before GBCAs are used. In cases when MRI is deemed unavoidable, macrocyclic GBCAs or newer linear GBCAs may be used. The implementation of renal function assessment prior to MRI, and greater caution in the administration of GBCAs, significantly reduces the incidence of NSF [23].

Discussions concerning GBCA safety intensified following the reports of gadolinium residues accumulating in the brain. The retrospective study by Kanda et al. published in 2014 indicated a significant correlation between high signal intensities on non-enhanced T1-weighted images (T1WI) in the globus pallidus (GB) and dentate nucleus (DN) and linear GBCA administration in patients with normal renal and liver function [24]. Moreover, a correlation between signal intensity and the quantity of GBCA dosage was proved [24]. Many scientific studies are now available in animal, paediatric and adult populations indicating a correlation between linear GBCA exposure and hyperintensities on non-enhanced T1WI in deep grey matter structures of the brain. In fact, the GB and the DN are the most commonly affected [25-29].

Studies considering a correlation between macrocyclic GBCAs and changes in MRI signal intensity have been few in number, and their conclusions are unclear. Most published papers indicate a lack of association between macrocyclic GBCAs and MRI changes [30-33]. One study that demonstrated a correlation between macrocyclic GBCAs and hyperintensities in MRI was that by Stojanov et al., but this was compromised due to a methodological defect (uncertainty regarding previous linear GBCA exposure) [34]. Next came the study by Bjørnerud et al. in which subjects had underlying disease of high-grade glioma, and their previous treatment with radiotherapy could potentially modify the obtained data [35].

T1WI MRI hyperintensity is not a characteristic of gadolinium accumulation, and may be caused by the accumulation of other metals (e.g. iron, copper, manganese), a history of brain irradiation, neurofibromatosis type 1, Rendu-Osler-Weber disease, or Wilson disease [36, 37]. Post mortem studies are vital to prove gadolinium accumulation. Autopsy results, carried out on both animal and human models, have shown a strong correlation between T1 hyperintensity and gadolinium residues concentration with the use of ICP-MS method. This study technique allowed the confirmation of both linear and macrocyclic agents gadolinium residues deposition in the brain [38-40]. Furthermore, gadolinium compounds can deposit not only in deep grey matter but also in the cerebral cortex. Even one single-use dosage of a GBCA can lead to long-term gadolinium accumulation [41, 42].

\section{GBCAs penetration mechanism}

GBCAs cannot directly penetrate an intact BBB [43]. The study by Jost et al., based on rat cerebrospinal fluid (CSF) analysis using the fluid-attenuated inversion recovery (FLAIR) method, documented the enhancement of CSF signal after the administration of either linear or macrocyclic GBCAs [44]. Recently, this was confirmed in a clinical study where gadolinium was detected in the CSF even in patients with a presumably intact $\mathrm{BBB}$ [45].

All these studies suggest that CSF is a potential gateway for GBCA entry into brain tissues. These results have raised the question of a correlation between BBB damage and the amount of gadolinium residues accumulated in the brain. In 2019, Jost et al. proved in an animal model that BBB damage does not increase the amount of gadolinium retention [46].

\section{Clearance of gadolinium from the brain}

The GBCA clearance process from the human body is not yet fully understood. Smith et al. were the first to describe a reduction of gadolinium concentration, of about 50\%, 20 weeks 
after multiple linear GBCAs administration [47]. However, these results have been called into question by more recent studies. Linear GBCAs probably undergo partial dechelation and a large portion of gadolinium is retained in the brain, with binding of soluble gadolinium to macromolecules, making clearance potentially impossible. On the other hand, macrocyclic compounds are retained as original chelates and are eliminated over time $[42,48,49]$.

\section{Toxicity of gadolinium deposition}

The long-term effect of gadolinium accumulation in brain structures is unclear and still the subject of intensive research. Earlier publications did not find a correlation between the most frequently affected brain structures (GP, DN) and Parkinson's Disease and cerebellar syndrome $[50,51]$.

Multiple Sclerosis (MS) patients, due to constant disease surveillance, are the most exposed to potential GBCA toxicity. In patients with early MS, the signal hyperintensity observed in the GP, DN and thalamus is associated with lifetime cumulative gadodiamide administration without clinical or radiological correlates of disease progression [52]. Furthermore, gadolinium retention in brain structures of MS patients is not correlated with their clinical deterioration expressed in the form of the Expanded Disability Status Scale (EDSS) [53]. However, not all studies support the hypothesis that gadolinium retention does not cause toxicity. An 18-year observation of MS patients demonstrated a possible correlation between a decrease in verbal fluency scores in connection with mainly DN hyperintensity [54]. The latest study, using a new method for simultaneous T1 and T2 relaxometry in a prospective cohort of patients with MS and healthy controls, indicates that previous linear (but not macrocyclic) GBCAs administration is associated with higher relaxation rates in a dose-dependent manner. Higher relaxation in some regions is associated with cognitive impairment, but not physical disability or fatigue in MS [55]. The lack of clarity of the obtained results, in conjunction with the basic MS characteristics, does not allow unequivocal conclusions to be drawn but, due to repeated GBCA exposure, this group of patients should be closely monitored.

The preclinical study by Bower et al. performed on a basal ganglia neurons model yielded an interesting result. The authors evaluated cell death and several parameters of mitochondrial function. They demonstrated that increased GBCA toxicity on mitochondrial respiratory function and cell viability is correlated with an increase of GBCA concentration and a decrease in its kinetic stability [56].

A number of studies carried out in animal models are available, but their results are also inconclusive. After 20 weeks from linear administration to a group of healthy rats, neurotoxicity was not detectable [47]. Moreover, an analysis of chosen metabolic markers (cellular homeostasis, excitatory neurotransmitter, and metabolites specific to a cellular compartment) with $1 \mathrm{H}$-magnetic resonance spectroscopy (1H-MRS) and laser ablation ICP-MS revealed no significant metabolism and histological changes between gadodiamide and a control group [57]. On the other hand, some studies have found relevant alterations after GBCA administration in animal models. Murine macrophages exposure to gadolinium agents, even at low concentrations, induces mitochondrial stress and the production of inflammatory cytokines [58]. A recent study on experimental autoimmune encephalomyelitis revealed that after the repeated usage of linear GBCAs ongoing inflammation may facilitate the retention of gadolinium in the brain tissue. Therefore, it is possible that patients with chronic neuroinflammatory disorders form a group with a higher risk of the accumulation of gadolinium in brain structures [59].

\section{Alternative non-contrast-enhanced techniques of MRI}

Recent reports of gadolinium deposition in the brain structures have forced a modification in the recommendations regarding the use of GBCAs in clinical practice. According to these, GBCAs should only be used when absolutely necessary or in research settings with the appropriate guidance of protocols [60]. Therefore, efforts are ongoing to find new NCE methods which could potentially replace CE-MRI [61].

Arterial spin labelling (ASL) allows an estimation of brain perfusion [62]. It is possible that ASL could replace GBCA-based perfusion techniques such as dynamic contrast-enhanced (DCE) MRI. The ASL technique magnetically labels water protons in the blood by exposure to a radio-frequency pulse. Afterwards, these protons are transported by the blood to the organ of interest and incorporated into the tissue. With ASL, the imaging is performed twice, first without labelling (as a control) and then with labelling [63]. Recent studies have shown that ASL could be used to depict arteriovenous malformations, and assess dural arteriovenous fistulas and the reperfusion process after an acute ischaemic stroke, which is strongly associated with clinical outcome $[62,64]$.

Another MRI technique that may be useful in the evaluation of brain perfusion is Intravoxel Incoherent Motion (IVIM) imaging. IVIM enables simultaneous evaluation of diffusion and perfusion through a multi-b-value diffusion-weighted MRI acquisition [65]. Recent work in neuro-oncology has shown the potential for IVIM in differentiating tumour recurrence from post-treatment effects in the preoperative evaluation of tumour grade, and in differentiating primary CNS lymphoma from glioblastoma [66-68]. IVIM has also been applied in the setting of acute strokes, where perfusion imaging without gadolinium is of particular interest [69].

Time of flight (TOF) MRI is an NCE method which measures and depicts the bloodflow inside a vessel compared to the surrounding static tissue [70]. The TOF MRI technique can be used or imaging veins and arteries, and therefore could be used when evaluating for vessel patency in suspected occlusion or 
stenosis. However, TOF MRI is proven to be less effective in the evaluation of intracranial arterial occlusion than CE-MRI [71]. Hyperintense lesions in T1 images, such as lipomas, can be mistaken in TOF imaging for a vascular structure such as an aneurysm, although fat-saturated sequences can help distinguish between these conditions [72].

Phase contrast imaging (PC) is another MRI technique that can be used to visualise moving fluids. The PC technique derives contrast between flowing blood or CSF and stationary tissue by using the movement of transverse magnetisation to produce image contrast [73]. Multiple applications of phase-contrast imaging are actively used in clinical practice. In neurological imaging, phase-contrast imaging can be used to measure the flow of cerebrospinal fluid or to visualise arterial and venous flow [74].

We should mention here a recent prospective study by Christensen et al. in which the authors proposed a different approach to reducing GBCA enhancement in MRI. Their paper presents a comparison of Tmax values between full and half dose gadolinium perfusion studies in acute ischaemic stroke patients. Despite a somewhat limited study group, the authors were able to show high Tmax correspondence with similar appearance and numerical values of the calculated Tmax maps, indicating that half a dose may be a good solution. The viability of this proposed modification should however be confirmed in a larger study group [75].

Another extensively studied NCE technique is diffusion-weighted imaging (DWI), which depicts the diffusion of protons in the tissue. In diffusion MRI, tissues with restricted proton movement will appear bright with a low apparent diffusion coefficient (ADC). Advanced DWI techniques will allow visualisation of the movement of protons along white matter tracts or the estimation of perfusion metrics [61]. In particular, diffusion tensor imaging (DTI) may be used to map and characterise the three-dimensional diffusion of water as a function of spatial location. Diffusion methods can be used to quantify the changes in tissue microstructure induced by pathological conditions. Perfusion imaging has been shown to be helpful in the diagnostics of such conditions as strokes and tumours [76,77]. Both DWI and DTI have been tested as a potential contrast MRI replacement in MS patients. However, available results indicate that these techniques can only be used as supporting methods to CE-MRI [78-80].

A promising alternative to GBCA-enhanced MRI could be the use of magnetic resonance elastography (MRE) which can provide extensive information about the mechanical properties of studied tissues by analysing their response to oscillatory shear stress. This is still an experimental technique. Nevertheless, studies conducted in both MS patients and animal model experimental autoimmune encephalomyelitis (EAE) have proved that inflammation was associated with a reduction of brain stiffness $[81,82]$. The research of Bigot et al. performed on a relapsing-remitting EAE model shows that a reduction of brain stiffness correlates with clinical disability and is associated with enhanced expression of the extracellular matrix protein fibronectin. These results taken together suggest that MRE could potentially emerge as a safe tool to monitor MS activity [83].

MR spectroscopy (MRS) is a helpful imaging method used for differential diagnosis and treatment effect monitoring, providing information about the chemical composition of studied tissues. This method addresses metabolic pathways and their steady states in different tissue types. The brain has been one of the tissues most studied by MRS. Although 31P-MRS is of outstanding efficacy in the evaluation of sources of metabolic energy in the brain, $1 \mathrm{H}$-MRS has become the major clinically applied method in neurospectroscopy, as it provides information on markers of neuronal function, myelin, cell membranes, and metabolic active compounds [84]. None of the CE-MRI methods can assess tissue properties in the way that MRS can, and therefore this method could be useful for tumour characterisation and assessing the results of treatment [85].

Yet another NCE technique, susceptibility weighted imaging (SWI), can be used to depict small areas in the brain causing heterogenicity in the magnetic field. Similarly to CET1WI, SWI carries the advantage of being able to depict tiny structures with a high lesion-to-background signal. The SWI technique is useful in detecting cerebral microbleeds, iron deposition, and cerebral calcifications [86].

The last, but not least, NCE technique is amide proton transfer (APT) imaging, which was developed to assess tissue $\mathrm{pH}$ and protein content by MRI [87]. With this method the characterisation of tissue properties could be an alternative to CE-T1WI depiction of impaired BBB [88]. APT can be used, among others, in brain tumour assessment. However, proteinaceous cysts or haemorrhages can also increase the signal compared to high-grade tumour components [89].

\section{New GBCAs and alternative MRI contrast agents}

The lack of clinically safe contrast agents in the light of GBCA's retention and possible neurotoxicity has significantly intensified research into alternative compounds.

Today, the only action a clinician can take to reduce patient exposure to GBCAs is to reduce the administered dose. One possible method of achieving such a reduction, without losing the quality of the obtained images, is analysis using the deep brain method. It is possible that machine learning could lead to a 10 -fold reduction in the required contrast dose [90]. Recently published results of a phase IIb clinical study on the new high relaxivity macrocyclic GBCA gadopiclenol suggest that a reduced dose of gadopiclenol, compared to clinically used dosages, is possible without a reduction in CNS image quality [91]. Moreover, there are attempts to improve GBCAs by replacing the standard chelate agents with carbon nanomaterials. This new T1-enhancing contrast agent class is characterised by far greater proton relaxivity (up to 90 -fold) 
which allows the attainment of reliable images with a lower dose. Carbon nanomaterials are also capable of translocating across membranes and label cells. Their modification with the use of biopolymers (DNA, RNA, proteins) causes them to have contrast selectivity towards cells and tissues. The potential of multifunctionality encompasses multimodal imaging and the combination of imaging and therapy [92].

Another extensively studied class of contrast agents are compounds containing in their structure a metal other than gadolinium. One of these agents is manganese-based $\mathrm{Mn}-\mathrm{PyC} 3 \mathrm{~A}$ which is characterised by a relaxivity comparable to GBCAs. It is cleared via both renal and hepatobiliary excretion and is eliminated from blood plasma. Furthermore, a liquid chromatography examination of blood plasma and urine reveals that $\mathrm{Mn}-\mathrm{PyC} 3 \mathrm{~A}$ is cleared intact, without undergoing metabolism or degradation [93]. Manganese containing mangafodipir, which is no longer authorised by the European Medicines Agency for liver imaging, was recently used in a group of healthy volunteers to evaluate the dynamics of manganese enhancement of the brain. The results of this study indicate a successful visualisation of intra- and extracranial structures that lie outside the BBB without adverse clinical effects [94, 95].

Promising results have been obtained in studies of contrast agents containing iron instead of gadolinium. This class includes iron-based contrast agents (IBCA) and superparamagnetic iron oxide nanoparticles (SPIONs). In the case of IBCA, two main challenges need to be confronted before they can be used in clinical practice. Firstly, iron chelates provide far less signal than gadolinium chelates. Secondly, iron chelates can be toxic at high doses, which can manifest in the ability to catalyse oxidative Fenton chemistry to produce hydroxyl radicals. Studies indicate that both of these challenges can be addressed with a proper chelate structure design. Recent research by Bales et al. has proposed using the Fe-HBED analogues which, compared to the parent Fe-HBED, have lower serum protein binding and higher relaxivity than do a representative GBCA [96].

SPIONs are excellent MR contrast agents which possess unique magnetic properties with strong shortening effects under longitudinal and transverse relaxation. The coating of SPIONs with polymers (PVP, PEG, PVA, Dextran) prevents their aggregation and limits their toxic effect on body tissues, and this allows for their medical applications. Many iron oxide nanoparticles have been evaluated in preclinical and clinical trials, and several of them have reached the market. However, some of the approved SPIONs have later been withdrawn [97].

A different approach is metal-free contrast agents with organic radical contrast agents (ORCAs) based on paramagnetic aminoxyl moieties. These are characterised by low cytotoxicity and high biodegradability. Compared to
GBCAs, they have low relaxivity and are reduced rapidly to MRI-silent hydroxylamines in physiological conditions. In order to improve their efficiency, the attachment of supramolecular and biomacromolecular is needed [98].

The general characteristics of the abovementioned alternative contrast agents are set out in Table 2 .

\section{Conclusions}

The utility of GBCAs in the initial diagnosis and monitoring of numerous diseases is indisputable. Nevertheless, many studies have confirmed gadolinium retention in deep brain structures, especially in the GP and DN and recently in the cerebral cortex. It has been proved that, due to their biochemical structure, linear nonionic GBCAs demonstrate the greatest susceptibility to dechelation. Therefore, they are responsible for most gadolinium retention in the CNS. On the other hand, histopathological studies have indicated that macrocyclic GBCAs are likewise accumulated in brain tissues but in significantly lower concentrations, which may explain why after repeated macrocyclic GBCAs administration, no intensity change in MRI has been observed.

Based on this study, the European Medicines Agency has published restrictions on the use of linear gadolinium agents in body scans. As a result, the marketing authorisations for intravenous linear agents other than gadoxetic acid and gadobenic acid have been suspended in the European Union. Macrocyclic agents were recommended due to their greater stability and lesser tendency to gadolinium release. Healthcare professionals should only use gadolinium contrast agents when necessary, and with the lowest agent dose possible [99].

This suspension, and the possible toxicity of GBCAs, has led to an intensification of studies into alternative CE-imaging methods and contrast agents. The most highly exposed group of neurological patients to the adverse effects of GBCAs are MS patients because of the need for frequent MRI check-ups to assess disease activity. Future research should aim to determine whether taking into account additional features of MS pathogenesis elucidated by NC-MRI techniques can increase the accuracy of MS diagnosis.

The unclear effects of the toxicity of gadolinium retention in the brain structures and the lack of dependable neurological symptoms occurrence, and long-term risk data, should propel healthcare professionals into a thorough analysis of the risk/benefits ratio of GBCAs administration, especially in patients exposed to repeated contrast agent administration. We recommend the scientific literature be monitored in terms of subsequent publications on GBCAs, and their potential substitution by alternative agents or diagnostic methods.

Acknowledgments: The authors thank Professor Jerzy Walecki for his invaluable comments on the manuscript. 


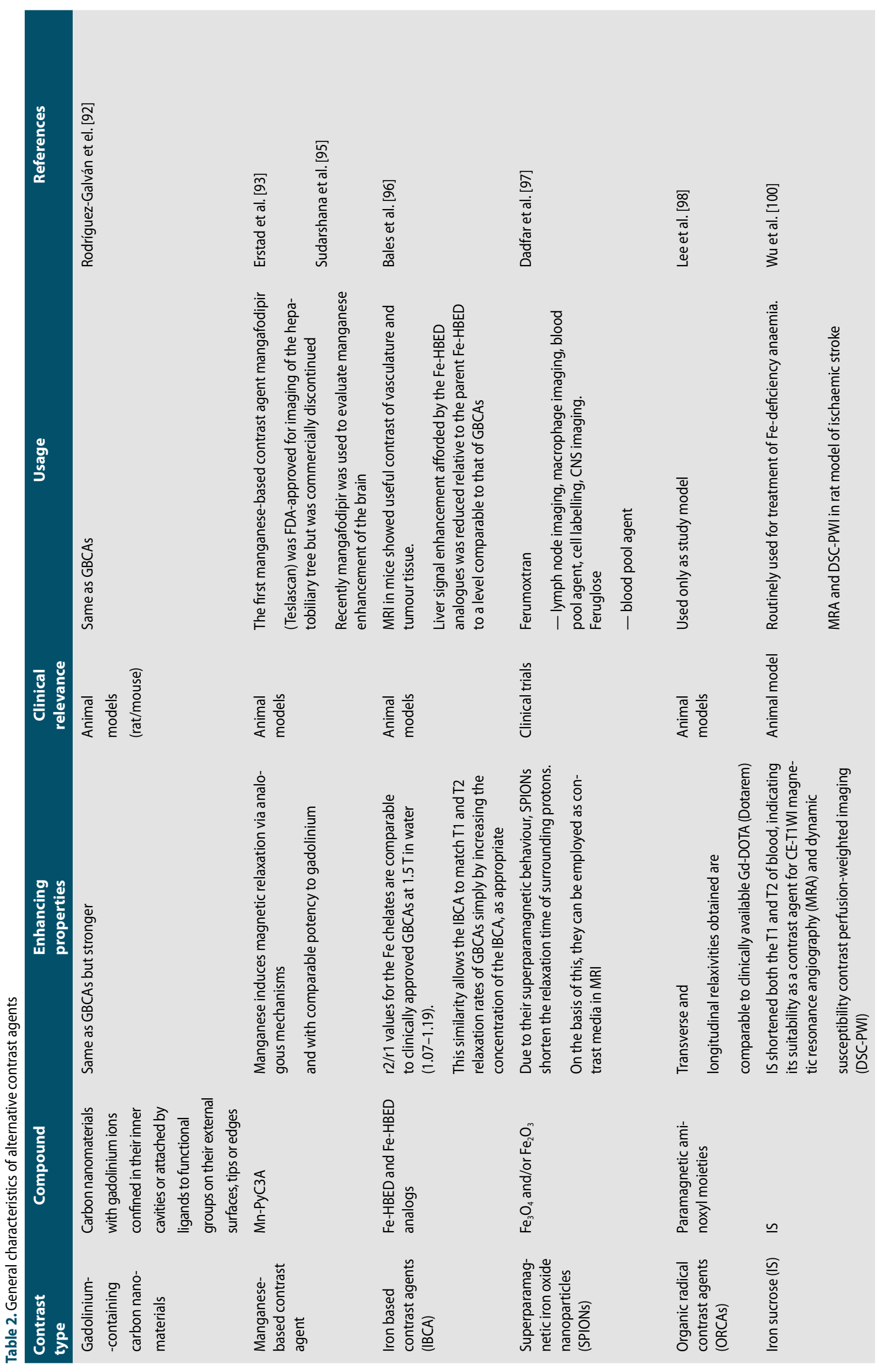




\section{References}

1. Almutairi A, Mahmud R, Suppiah S, et al. Accuracy of MRI Sequences in Detecting Multiple Sclerosis (MS) Lesions: A Systematic Review. Advances in Bioscience and Clinical Medicine. 2019; 7(2): 39, doi: 10.7575/aiac.abcmed.v.7n.2p.39.

2. Bhargava R, Hahn G, Hirsch W, et al. Contrast-enhanced magnetic resonance imaging in pediatric patients: review and recommendations for current practice. Magn Reson Insights. 2013; 6: 95-111, doi: 10.4137/MRI.S12561, indexed in Pubmed: 25114547.

3. Lohrke J, Frenzel T, Endrikat J, et al. 25 Years of Contrast-Enhanced MRI: Developments, Current Challenges and Future Perspectives. Adv Ther. 2016; 33(1): 1-28, doi: 10.1007/s12325-015-0275-4, indexed in Pubmed: 26809251.

4. Caravan P, Ellison JJ, McMurry TJ, et al. Gadolinium(III) Chelates as MRI Contrast Agents: Structure, Dynamics, and Applications. Chem Rev. 1999; 99(9): 2293-2352, doi: 10.1021/cr980440x, indexed in Pubmed: 11749483.

5. Lauffer R. Paramagnetic metal complexes as water proton relaxation agents for NMR imaging: theory and design. Chemical Reviews. 1987; 87(5): 901-927, doi: 10.1021/cr00081a003.

6. Essig M, Anzalone N, Combs SE, et al. MR imaging of neoplastic central nervous system lesions: review and recommendations for current practice. AJNR Am J Neuroradiol. 2012; 33(5): 803-817, doi: 10.3174/ajnr.A2640, indexed in Pubmed: 22016411.

7. Fraum TJ, Ludwig DR, Bashir MR, et al. Gadolinium-based contrast agents: A comprehensive risk assessment. J Magn Reson Imaging. 2017; 46(2): 338-353, doi: 10.1002/jmri.25625, indexed in Pubmed: 28083913.

8. Runge VM. Safety of the Gadolinium-Based Contrast Agents for Magnetic Resonance Imaging, Focusing in Part on Their Accumulation in the Brain and Especially the Dentate Nucleus. Invest Radiol. 2016; 51(5): 273-279, doi: 10.1097/RLI.00000000000000273, indexed in Pubmed: 26945278.

9. Rogosnitzky M, Branch S. Gadolinium-based contrast agent toxicity: a review of known and proposed mechanisms. Biometals. 2016; 29(3): 365-376, doi: 10.1007/s10534-016-9931-7, indexed in Pubmed: 27053146.

10. Kanal E, Maravilla K, Rowley HA. Gadolinium contrast agents for CNS imaging: current concepts and clinical evidence. AJNR Am J Neuroradiol. 2014; 35(12): 2215-2226, doi: 10.3174/ajnr.A3917, indexed in Pubmed: 24852287.

11. Bellin MF, Van Der Molen AJ. Extracellular gadolinium-based contrast media: an overview. Eur J Radiol. 2008; 66(2): 160-167, doi: 10.1016/j.ejrad.2008.01.023, indexed in Pubmed: 18358659.

12. Port M, Idée JM, Medina C, et al. Efficiency, thermodynamic and kinetic stability of marketed gadolinium chelates and their possible clinical consequences: a critical review. Biometals. 2008; 21(4): 469-490, doi: 10.1007/s10534-008-9135-x, indexed in Pubmed: 18344005.

13. Dillman JR, Ellis JH, Cohan RH, et al. Frequency and severity of acute allergic-like reactions to gadolinium-containing i.v. contrast media in children and adults. AJR Am J Roentgenol. 2007; 189(6): 1533-1538, doi: 10.2214/AJR.07.2554, indexed in Pubmed: 18029897.

14. Ramalho M, Ramalho J. Gadolinium-Based Contrast Agents: Associated Adverse Reactions. Magn Reson Imaging Clin N Am. 2017; 25(4): 755-764, doi: 10.1016/j.mric.2017.06.006, indexed in Pubmed: 28964465.
15. ACR Manual On Contrast Media 2020 ACR Committee on Drugs and Contrast Media. ; 2020.

16. Nelson KL, Gifford LM, Lauber-Huber C, et al. Clinical safety of gadopentetate dimeglumine. Radiology. 1995; 196(2): 439-443, doi: 10.1148/radiology.196.2.7617858, indexed in Pubmed: 7617858.

17. Behzadi AH, Zhao Y, Farooq Z, et al. Immediate Allergic Reactions to Gadolinium-based Contrast Agents: A Systematic Review and Meta-Analysis. Radiology. 2018; 286(2): 471-482, doi: 10.1148/ radiol.2017162740, indexed in Pubmed: 28846495.

18. ESUR Contrast Media Safety Guidelines. http://www.esur.org/esur-guidelines/ (10.2019).

19. Tweedle MF, Wedeking P, Kumar K. Biodistribution of radiolabeled, formulated gadopentetate, gadoteridol, gadoterate, and gadodiamide in mice and rats. Invest Radiol. 1995; 30(6): 372-380, doi: 10.1097/00004424-199506000-00008, indexed in Pubmed: 7490190.

20. Gibby WA, Gibby KA, Gibby WA. Comparison of Gd DTPA-BMA (Omniscan) versus Gd HP-D03A (ProHance) retention in human bone tissue by inductively coupled plasma atomic emission spectroscopy. Invest Radiol. 2004; 39(3): 138-142, doi: 10.1097/01. rli.0000112789.57341.01, indexed in Pubmed: 15076005.

21. White GW, Gibby WA, Tweedle MF. Comparison of Gd(DTPA-BMA) (Omniscan) versus Gd(HP-D03A) (ProHance) relative to gadolinium retention in human bone tissue by inductively coupled plasma mass spectroscopy. Invest Radiol. 2006; 41(3): 272-278, doi: 10.1097/01. rli.0000186569.32408.95, indexed in Pubmed: 16481910.

22. Grobner T. Gadolinium--a specific trigger for the development of nephrogenic fibrosing dermopathy and nephrogenic systemic fibrosis? Nephrol Dial Transplant. 2006; 21(4): 1104-1108, doi: 10.1093/ndt/ gfk062, indexed in Pubmed: 16431890.

23. Schieda N, Blaichman Jl, Costa AF, et al. Gadolinium-Based Contrast Agents in Kidney Disease: Comprehensive Review and Clinical Practice Guideline Issued by the Canadian Association of Radiologists. Can Assoc Radiol J. 2018; 69(2): 136-150, doi: 10.1016/j. carj.2017.11.002, indexed in Pubmed: 29706252.

24. Kanda T, Ishii K, Kawaguchi $\mathrm{H}$, et al. High signal intensity in the dentate nucleus and globus pallidus on unenhanced T1-weighted MR images: relationship with increasing cumulative dose of a gadolinium-based contrast material. Radiology. 2014; 270(3): 834-841, doi: 10.1148/radiol.13131669, indexed in Pubmed: 24475844.

25. Errante $\mathrm{Y}$, Cirimele V, Mallio CA, et al. Progressive increase of $\mathrm{T} 1$ signal intensity of the dentate nucleus on unenhanced magnetic resonance images is associated with cumulative doses of intravenously administered gadodiamide in patients with normal renal function, suggesting dechelation. Invest Radiol. 2014; 49(10): 685-690, doi: 10.1097/ RLI.0000000000000072, indexed in Pubmed: 24872007.

26. Ramalho J, Semelka RC, AlObaidy M, et al. Signal intensity change on unenhanced T1-weighted images in dentate nucleus following gadobenate dimeglumine in patients with and without previous multiple administrations of gadodiamide. Eur Radiol. 2016; 26(11): 4080-4088, doi: 10.1007/s00330-016-4269-7, indexed in Pubmed: 26911888.

27. Roberts DR, Holden KR. Progressive increase of T1 signal intensity in the dentate nucleus and globus pallidus on unenhanced T1-weighted MR images in the pediatric brain exposed to multiple doses of gadolinium contrast. Brain Dev. 2016; 38(3): 331-336, doi: 10.1016/j. braindev.2015.08.009, indexed in Pubmed: 26345358.

28. Flood TF, Stence NV, Maloney JA, et al. Pediatric Brain: Repeated Exposure to Linear Gadolinium-based Contrast Material Is Associated with Increased Signal Intensity at Unenhanced T1-weighted 
MR Imaging. Radiology. 2017; 282(1): 222-228, doi: 10.1148/radiol.2016160356, indexed in Pubmed: 27467467.

29. Robert P, Violas X, Grand S, et al. Linear Gadolinium-Based Contrast Agents Are Associated With Brain Gadolinium Retention in Healthy Rats. Invest Radiol. 2016; 51(2): 73-82, doi: 10.1097/ RLI.0000000000000241, indexed in Pubmed: 26606549.

30. Kanda T, Osawa M, Oba H, et al. High Signal Intensity in Dentate Nucleus on Unenhanced T1-weighted MR Images: Association with Linear versus Macrocyclic Gadolinium Chelate Administration. Radiology. 2015; 275(3): 803-809, doi: 10.1148/radiol.14140364, indexed in Pubmed: 25633504.

31. Radbruch A, Weberling LD, Kieslich PJ, et al. Gadolinium retention in the dentate nucleus and globus pallidus is dependent on the class of contrast agent. Radiology. 2015; 275(3): 783-791, doi: 10.1148/ radiol.2015150337, indexed in Pubmed: 25848905.

32. Radbruch A, Haase R, Kickingereder P, et al. Pediatric Brain: No Increased Signal Intensity in the Dentate Nucleus on Unenhanced T1-weighted MR Images after Consecutive Exposure to a Macrocyclic Gadolinium-based Contrast Agent. Radiology. 2017; 283(3): 828-836, doi: 10.1148/radiol.2017162980, indexed in Pubmed: 28273007.

33. Pozeg P, Forget J, Meuli RA, et al. Age, But Not Repeated Exposure to Gadoterate Meglumine, Is Associated With T1- and T2-Weighted Signal Intensity Changes in the Deep Brain Nuclei of Pediatric Patients. Invest Radiol. 2019; 54(9): 537-548, doi: 10.1097/ RLI.0000000000000564, indexed in Pubmed: 30973458.

34. Agris J, Pietsch H, Balzer T. What Evidence Is There That Gadobutrol Causes Increasing Signal Intensity within the Dentate Nucleus and Globus Pallidus on Unenhanced T1W MRI in Patients with RRMS? Eur Radiol. 2016; 26(3): 816-817, doi: 10.1007/s00330-015-4019-2, indexed in Pubmed: 26391362.

35. Bjørnerud A, Vatnehol SA, Larsson C, et al. Signal Enhancement of the Dentate Nucleus at Unenhanced MR Imaging after Very High Cumulative Doses of the Macrocyclic Gadolinium-based Contrast Agent Gadobutrol: An Observational Study. Radiology. 2017; 285(2): 434-444, doi: 10.1148/radiol.2017170391, indexed in Pubmed: 28885891.

36. Kanda T, Nakai Y, Aoki S, et al. Contribution of metals to brain MR signal intensity: review articles. Jpn J Radiol. 2016; 34(4): 258-266, doi: 10.1007/s11604-016-0532-8, indexed in Pubmed: 26932404.

37. Kasahara S, Miki Y, Kanagaki M, et al. Hyperintense dentate nucleus on unenhanced T1-weighted MR images is associated with a history of brain irradiation. Radiology. 2011; 258(1): 222-228, doi: 10.1148/ radiol.10100508, indexed in Pubmed: 21045180.

38. Lohrke J, Frisk AL, Frenzel T, et al. Histology and Gadolinium Distribution in the Rodent Brain After the Administration of Cumulative High Doses of Linear and Macrocyclic Gadolinium-Based Contrast Agents. Invest Radiol. 2017; 52(6): 324-333, doi: 10.1097/ RLI.0000000000000344, indexed in Pubmed: 28323657.

39. McDonald RJ, McDonald JS, Dai D, et al. Comparison of Gadolinium Concentrations within Multiple Rat Organs after Intravenous Administration of Linear versus Macrocyclic Gadolinium Chelates. Radiology. 2017; 285(2): 536-545, doi: 10.1148/radiol.2017161594, indexed in Pubmed: 28640692.

40. Gianolio E, Bardini P, Arena F, et al. Gadolinium Retention in the Rat Brain: Assessment of the Amounts of Insoluble Gadolinium-containing Species and Intact Gadolinium Complexes after Repeated Administration of Gadolinium-based Contrast Agents. Radiology. 2017; 285(3): 839-849, doi: 10.1148/radiol.2017162857, indexed in Pubmed: 28873047.
41. Minaeva O, Hua N, Franz ES, et al. Nonhomogeneous Gadolinium Retention in the Cerebral Cortex after Intravenous Administration of Gadolinium-based Contrast Agent in Rats and Humans. Radiology. 2020; 294(2): 377-385, doi: 10.1148/radiol.2019190461, indexed in Pubmed: 31769744.

42. Strzeminska I, Factor C, Robert P, et al. Long-Term Evaluation of Gadolinium Retention in Rat Brain After Single Injection of a Clinically Relevant Dose of Gadolinium-Based Contrast Agents. Invest Radiol. 2020; 55(3): 138-143, doi: 10.1097/RLI.0000000000000623, indexed in Pubmed: 31917763.

43. Weinmann HJ, Brasch RC, Press WR, et al. Characteristics of gadolinium-DTPA complex: a potential NMR contrast agent. AJR Am J Roentgenol. 1984; 142(3): 619-624, doi: 10.2214/ajr.142.3.619, indexed in Pubmed: 6607655.

44. Jost G, Frenzel T, Lohrke J, et al. Penetration and distribution of gadolinium-based contrast agents into the cerebrospinal fluid in healthy rats: a potential pathway of entry into the brain tissue. Eur Radiol. 2017; 27(7): 2877-2885, doi: 10.1007/s00330-016-4654-2, indexed in Pubmed: 27832312.

45. Berger F, Kubik-Huch RA, Niemann T, et al. Gadolinium Distribution in Cerebrospinal Fluid after Administration of a Gadolinium-based MR Contrast Agent in Humans. Radiology. 2018; 288(3): 703-709, doi: 10.1148/radiol.2018171829, indexed in Pubmed: 29737953.

46. Jost G, Frenzel T, Boyken J, et al. Impact of brain tumors and radiotherapy on the presence of gadolinium in the brain after repeated administration of gadolinium-based contrast agents: an experimental study in rats. Neuroradiology. 2019; 61(11): 1273-1280, doi: 10.1007/ s00234-019-02256-3, indexed in Pubmed: 31297571.

47. Smith APL, Marino M, Roberts J, et al. Clearance of Gadolinium from the Brain with No Pathologic Effect after Repeated Administration of Gadodiamide in Healthy Rats: An Analytical and Histologic Study. Radiology. 2017; 282(3): 743-751, doi: 10.1148/radiol.2016160905, indexed in Pubmed: 27673510.

48. Jost G, Frenzel T, Boyken J, et al. Long-term Excretion of Gadolinium-based Contrast Agents: Linear versus Macrocyclic Agents in an Experimental Rat Model. Radiology. 2019; 290(2): 340-348, doi: 10.1148/ radiol.2018180135, indexed in Pubmed: 30422091.

49. Robert $P$, Fingerhut $S$, Factor $C$, et al. One-year Retention of Gadolinium in the Brain: Comparison of Gadodiamide and Gadoterate Meglumine in a Rodent Model. Radiology. 2018; 288(2): 424-433, doi: 10.1148/radiol.2018172746, indexed in Pubmed: 29786486.

50. Welk B, McArthur E, Morrow SA, et al. Association Between Gadolinium Contrast Exposure and the Risk of Parkinsonism. JAMA. 2016; 316(1): 96-98, doi: 10.1001/jama.2016.8096, indexed in Pubmed: 27380348.

51. Perrotta G, Metens T, Absil J, et al. Absence of clinical cerebellar syndrome after serial injections of more than 20 doses of gadoterate, a macrocyclic GBCA: a monocenter retrospective study. J Neurol. 2017; 264(11): 2277-2283, doi: 10.1007/s00415-017-8631-8, indexed in Pubmed: 28956156.

52. Zivadinov R, Bergsland N, Hagemeier J, et al. Cumulative gadodiamide administration leads to brain gadolinium deposition in early MS. Neurology. 2019; 93(6): e611-e623, doi: 10.1212/ WNL.0000000000007892, indexed in Pubmed: 31285398.

53. Cocozza S, Pontillo G, Lanzillo R, et al. MRI features suggestive of gadolinium retention do not correlate with Expanded Disability Status Scale worsening in Multiple Sclerosis. Neuroradiology. 2019; 61(2): 
155-162, doi: 10.1007/s00234-018-02150-4, indexed in Pubmed: 30617409.

54. Forslin Y, Shams S, Hashim F, et al. Retention of Gadolinium-Based Contrast Agents in Multiple Sclerosis: Retrospective Analysis of an 18Year Longitudinal Study. AJNR Am J Neuroradiol. 2017; 38(7): 13111316, doi: 10.3174/ajnr.A5211, indexed in Pubmed: 28495943.

55. Forslin Y, Martola J, Bergendal $\AA$, et al. Gadolinium Retention in the Brain: An MRI Relaxometry Study of Linear and Macrocyclic Gadolinium-Based Contrast Agents in Multiple Sclerosis. AJNR Am J Neuroradiol. 2019; 40(8): 1265-1273, doi: 10.3174/ajnr.A6112, indexed in Pubmed: 31248867.

56. Bower DV, Richter JK, von Tengg-Kobligk H, et al. Gadolinium-Based MRI Contrast Agents Induce Mitochondrial Toxicity and Cell Death in Human Neurons, and Toxicity Increases With Reduced Kinetic Stability of the Agent. Invest Radiol. 2019; 54(8): 453-463, doi: 10.1097/ RLI.0000000000000567, indexed in Pubmed: 31265439.

57. El Hamrani D, Vives V, Buchholz R, et al. Effect of Long-Term Retention of Gadolinium on Metabolism of Deep Cerebellar Nuclei After Repeated Injections of Gadodiamide in Rats. Invest Radiol. 2020; 55(2): 120-128, doi: 10.1097/RLI.0000000000000621, indexed in Pubmed: 31876627.

58. Weng Tl, Chen HJ, Lu CW, et al. Exposure of Macrophages to Low-Dose Gadolinium-Based Contrast Medium: Impact on Oxidative Stress and Cytokines Production. Contrast Media Mol Imaging. 2018; 2018: 3535769, doi: 10.1155/2018/3535769, indexed in Pubmed: 30627059.

59. Wang S, Hesse B, Roman M, et al. Increased Retention of Gadolinium in the Inflamed Brain After Repeated Administration of Gadopentetate Dimeglumine: A Proof-of-Concept Study in Mice Combining ICP-MS and Micro- and Nano-SR-XRF. Invest Radiol. 2019; 54(10): 617-626, doi: 10.1097/RLI.0000000000000571, indexed in Pubmed: 31033673.

60. Gulani V, Calamante F, Shellock FG, et al. International Society for Magnetic Resonance in Medicine. Gadolinium deposition in the brain: summary of evidence and recommendations. Lancet Neurol. 2017; 16(7): 564-570, doi: 10.1016/S1474-4422(17)30158-8, indexed in Pubmed: 28653648.

61. Falk Delgado A, Van Westen D, Nilsson M, et al. Diagnostic value of alternative techniques to gadolinium-based contrast agents in MR neuroimaging-a comprehensive overview. Insights Imaging. 2019; 10(1): 84, doi: 10.1186/s13244-019-0771-1, indexed in Pubmed: 31444580 .

62. Haller S, Zaharchuk G, Thomas DL, et al. Arterial Spin Labeling Perfusion of the Brain: Emerging Clinical Applications. Radiology. 2016; 281(2): 337-356, doi: 10.1148/radiol.2016150789, indexed in Pubmed: 27755938.

63. Detre JA, Alsop DC, Vives LR, et al. Noninvasive MRI evaluation of cerebral blood flow in cerebrovascular disease. Neurology. 1998; 50(3): 633-641, doi: 10.1212/wnl.50.3.633, indexed in Pubmed: 9521248.

64. Blauwblomme T, Naggara 0, Brunelle F, et al. Arterial spin labeling magnetic resonance imaging: toward noninvasive diagnosis and follow-up of pediatric brain arteriovenous malformations. J Neurosurg Pediatr. 2015; 15(4): 451-458, doi: 10.3171/2014.9.PEDS14194, indexed in Pubmed: 25634818.

65. Le Bihan D, Breton E, Lallemand D, et al. MR imaging of intravoxel incoherent motions: application to diffusion and perfusion in neurologic disorders. Radiology. 1986; 161(2): 401-407, doi: 10.1148/ radiology.161.2.3763909, indexed in Pubmed: 3763909.

66. Kim DY, Kim HS, Goh MJ, et al. Utility of intravoxel incoherent motion MR imaging for distinguishing recurrent metastatic tumor from treat- ment effect following gamma knife radiosurgery: initial experience. AJNR Am J Neuroradiol. 2014; 35(11): 2082-2090, doi: 10.3174/ ajnr.A3995, indexed in Pubmed: 24970548.

67. Federau C, Meuli R, O'Brien K, et al. Perfusion measurement in brain gliomas with intravoxel incoherent motion MRI. AJNR Am J Neuroradiol. 2014; 35(2): 256-262, doi: 10.3174/ajnr.A3686, indexed in Pubmed: 23928134.

68. Suh $\mathrm{CH}$, Kim HoS, Lee SS, et al. Atypical imaging features of primary central nervous system lymphoma that mimics glioblastoma: utility of intravoxel incoherent motion MR imaging. Radiology. 2014; 272(2): 504-513, doi: 10.1148/radiol.14131895, indexed in Pubmed: 24697149.

69. Federau C, Sumer S, Becce F, et al. Intravoxel incoherent motion perfusion imaging in acute stroke: initial clinical experience. Neuroradiology. 2014; 56(8): 629-635, doi: 10.1007/s00234-014-1370-y, indexed in Pubmed: 24838807.

70. Nishimura DG, Macovski A, Pauly JM, et al. MR angiography by selective inversion recovery. Magn Reson Med. 1987; 4(2): 193-202, doi: 10.1002/mrm.1910040214, indexed in Pubmed: 3561250.

71. Dhundass S, Savatovsky J, Duron L, et al. Improved detection and characterization of arterial occlusion in acute ischemic stroke using contrast enhanced MRA. J Neuroradiol. 2020; 47(4): 278-283, doi: 10.1016/j.neurad.2019.02.011, indexed in Pubmed: 30853544.

72. Kemmling A, Noelte I, Gerigk L, et al. A diagnostic pitfall for intracranial aneurysms in time-of-flight MR angiography: small intracranial lipomas. AJR Am J Roentgenol. 2008; 190(1): W62-W67, doi: 10.2214/ AJR.07.2517, indexed in Pubmed: 18094274.

73. Saito Y. 8 Current Status of Magnetic Resonance Angiography. vol. ; 7: 2018.

74. Wymer DT, Patel KP, Burke WF, et al. Phase-Contrast MRI: Physics, Techniques, and Clinical Applications. Radiographics. 2020; 40(1): 122-140, doi: 10.1148/rg.2020190039, indexed in Pubmed: 31917664.

75. Christensen S, Amukotuwa S, Lansberg MG, et al. Comparison of Tmax values between full- and half-dose gadolinium perfusion studies. J Cereb Blood Flow Metab. 2020 [Epub ahead of print]: 271678X20914537, doi: 10.1177/0271678X20914537, indexed in Pubmed: 32208802.

76. Roberts T, Rowley H. Diffusion weighted magnetic resonance imaging in stroke. European Journal of Radiology. 2003; 45(3): 185-194, doi: 10.1016/s0720-048x(02)00305-4.

77. Yamashita K, Hiwatashi A, Togao 0 , et al. Diagnostic utility of intravoxel incoherent motion $\mathrm{mr}$ imaging in differentiating primary central nervous system lymphoma from glioblastoma multiforme. J Magn Reson Imaging. 2016; 44(5): 1256-1261, doi: 10.1002/jmri.25261, indexed in Pubmed: 27093558.

78. Gupta A, Al-Dasuqi K, Xia F, et al. The Use of Noncontrast Quantitative MRI to Detect Gadolinium-Enhancing Multiple Sclerosis Brain Lesions: A Systematic Review and Meta-Analysis. AJNR Am J Neuroradiol. 2017; 38(7): 1317-1322, doi: 10.3174/ajnr.A5209, indexed in Pubmed: 28522663.

79. Unal S, Peker E, Erdogan S, et al. Is It Possible to Discriminate Active MS Lesions with Diffusion Weighted Imaging? Eurasian J Med. 2019; 51(3): 219-223, doi: 10.5152/eurasianjmed.2019.18473, indexed in Pubmed: 31692763.

80. Arashloo FT, Hanzaei FF, Sedighi B, et al. Efficacy of diffusion-weighted imaging in symptomatic and asymptomatic multiple sclerotic plaques. J Family Med Prim Care. 2019; 8(7): 2409-2413, doi: 10.4103/jfmpc. jfmpc_420_19, indexed in Pubmed: 31463267. 
81. Wuerfel J, Paul F, Beierbach B, et al. MR-elastography reveals degradation of tissue integrity in multiple sclerosis. Neuroimage. 2010; 49(3): 2520-2525, doi: 10.1016/j.neuroimage.2009.06.018, indexed in Pubmed: 19539039.

82. Riek K, Millward JM, Hamann I, et al. Magnetic resonance elastography reveals altered brain viscoelasticity in experimental autoimmune encephalomyelitis. Neuroimage Clin. 2012; 1(1): 81-90, doi: 10.1016/j.nicl.2012.09.003, indexed in Pubmed: 24179740.

83. Bigot M, Chauveau F, Beuf O, et al. Magnetic Resonance Elastography of Rodent Brain. Front Neurol. 2018; 9: 1010, doi: 10.3389/ fneur.2018.01010, indexed in Pubmed: 30538670.

84. Burtscher IM, Holtås S. Proton MR spectroscopy in clinical routine. J Magn Reson Imaging. 2001; 13(4): 560-567, doi: 10.1002/ jmri.1079, indexed in Pubmed: 11276100.

85. Cianfoni A, Law M, Re TJ, et al. Clinical pitfalls related to short and long echo times in cerebral MR spectroscopy. Journal of Neuroradiology. 2011; 38(2): 69-75, doi: 10.1016/j.neurad.2010.10.001.

86. Liu S, Buch S, Chen Y, et al. Susceptibility-weighted imaging: current status and future directions. NMR in Biomedicine. 2016; 30(4): e3552, doi: 10.1002/nbm.3552.

87. Zhou J, Payen JF, Wilson DA, et al. Using the amide proton signals of intracellular proteins and peptides to detect $\mathrm{pH}$ effects in MRI. Nat Med. 2003; 9(8): 1085-1090, doi: 10.1038/nm907, indexed in Pubmed: 12872167.

88. Ward KM, Aletras AH, Balaban RS. A new class of contrast agents for MRI based on proton chemical exchange dependent saturation transfer (CEST). J Magn Reson. 2000; 143(1): 79-87, doi: 10.1006/ jmre.1999.1956, indexed in Pubmed: 10698648.

89. Kamimura K, Nakajo M, Yoneyama T, et al. Amide proton transfer imaging of tumors: theory, clinical applications, pitfalls, and future directions. Jpn J Radiol. 2019; 37(2): 109-116, doi: 10.1007/ s11604-018-0787-3, indexed in Pubmed: 30341472.

90. Gong E, Pauly JM, Wintermark M, et al. Deep learning enables reduced gadolinium dose for contrast-enhanced brain MRI. J Magn Reson Imaging. 2018; 48(2): 330-340, doi: 10.1002/jmri.25970, indexed in Pubmed: 29437269.

91. Bendszus M, Roberts D, Kolumban B, et al. Dose Finding Study of Gadopiclenol, a New Macrocyclic Contrast Agent, in MRI of Cen- tral Nervous System. Invest Radiol. 2020; 55(3): 129-137, doi: 10.1097/RLI.0000000000000624, indexed in Pubmed: 31917762.

92. Rodríguez-Galván A, Rivera M, García-López P, et al. Gadolinium-containing carbon nanomaterials for magnetic resonance imaging: Trends and challenges. J Cell Mol Med. 2020; 24(7): 3779-3794, doi: 10.1111/jcmm.15065, indexed in Pubmed: 32154648.

93. Erstad DJ, Ramsay IA, Jordan VC, et al. Tumor Contrast Enhancement and Whole-Body Elimination of the Manganese-Based Magnetic Resonance Imaging Contrast Agent Mn-PyC3A. Invest Radiol. 2019; 54(11): 697-703, doi: 10.1097/RLI.0000000000000593, indexed in Pubmed: 31356382.

94. European Medicines Agency. EMA/486286/2012 2012. https:// www.ema.europa.eu/en/documents/public-statement/public-statement-teslascan-withdrawal-marketing-authorisation-european-union_en.pdf.

95. Sudarshana DM, Nair G, Dwyer JT, et al. Manganese-Enhanced MRI of the Brain in Healthy Volunteers. AJNR Am J Neuroradiol. 2019; 40(8): 1309-1316, doi: 10.3174/ajnr.A6152, indexed in Pubmed: 31371354.

96. Bales BC, Grimmond B, Johnson BF, et al. Fe-HBED Analogs: A Promising Class of Iron-Chelate Contrast Agents for Magnetic Resonance Imaging. Contrast Media Mol Imaging. 2019; 2019: 8356931, doi: 10.1155/2019/8356931, indexed in Pubmed: 31969797.

97. Dadfar SM, Roemhild K, Drude NI, et al. Iron oxide nanoparticles: Diagnostic, therapeutic and theranostic applications. Adv Drug Deliv Rev. 2019; 138: 302-325, doi: 10.1016/j.addr.2019.01.005, indexed in Pubmed: 30639256.

98. Lee H, Shahrivarkevishahi A, Lumata JL, et al. Supramolecular and biomacromolecular enhancement of metal-free magnetic resonance imaging contrast agents. Chem Sci. 2020; 11(8): 2045-2050, doi: 10.1039/c9sc05510j, indexed in Pubmed: 32180926.

99. EMA's final opinion confirms restrictions on use of linear gadolinium agents in body scans | European Medicines Agency 2017. https:// www.ema.europa.eu/en/news/emas-final-opinion-confirms-restrictions-use-linear-gadolinium-agents-body-scans.

100. Wu S, Zhang H, Wang J, et al. Iron Sucrose as MRI Contrast Agent in Ischemic Stroke Model. J Magn Reson Imaging. 2020; 52(3): 836849, doi: 10.1002/jmri.27109, indexed in Pubmed: 32112623. 